\title{
ON LONG-RANGE DEPENDENCE IN REGENERATIVE PROCESSES BASED ON A GENERAL ON/OFF SCHEME
}

\author{
REMIGIJUS LEIPUS * AND \\ DONATAS SURGAILIS, ${ }^{* *}$ Vilnius University and Institute of Mathematics and Informatics, Vilnius
}

\begin{abstract}
In this paper, we obtain a closed form for the covariance function of a general stationary regenerative process. It is used to derive exact asymptotics of the covariance function of stationary ON/OFF and workload processes, when ON and OFF periods are heavy-tailed and mutually dependent. The case of a G/G/1/0 queueing system with heavy-tailed arrival and/or service times is studied in detail.
\end{abstract}

Keywords: Regenerative process; ON/OFF process; G/G/1/0 queue; long-range dependence

2000 Mathematics Subject Classification: Primary 60G10; 60K05; 62K25

\section{Introduction}

Willinger et al. (1997) introduced the notion of ON/OFF processes to model data traffic in communication networks. In their model, an idealized source alternates between two regimes: an $\mathrm{ON}$ state, in which it produces data at a constant rate, and an OFF state, in which it produces no data. It is usually assumed that consecutive ON and OFF durations are independent, having generally different distributions. The corresponding ON/OFF times form an alternating renewal process and the ON/OFF process $A(t)$ is equal to 1 if $t$ falls into an ON period and is equal to 0 if $t$ is in an OFF period. The primary aim of Willinger et al. (1997) and subsequent works on ON/OFF processes was the explanation and modelling of observed long-range dependence and self-similarity in network traffic. By a long-range dependence (LRD) property with rate $\alpha \in(0,1)$, we mean a regular decay of the autocovariance of a stationary process $X(t), t \in \mathbb{R}$, i.e.

$$
\operatorname{cov}(X(0), X(t))=L(t) t^{-\alpha}, \quad t>0,
$$

where $L$ is a function which is slowly varying at infinity.

In this paper, we obtain the LRD property of the stationary ON/OFF process $A(t)$ and the forward recurrence time $W(t)$ of the current busy period, in the case when the consecutive ON and OFF periods, $X^{\text {on }}$ and $Y^{\text {off }}$ respectively, are dependent. The random vectors $\left(X_{j}^{\text {on }}, Y_{j}^{\text {off }}\right)$, $j \geq 1$, are assumed to be independent and identically distributed (i.i.d.). It turns out that in this case, similarly to in the case of independent periods $X^{\text {on }}$ and $Y^{\text {off }}$ (see Heath et al. (1998)), the decay rate of the covariances of $A(t)$ and $W(t)$ is determined by the decay rate of tail probabilities of ON and OFF periods (see Corollary 3.2, below). In Section 4 we specify the

Received 18 May 2005; revision received 13 January 2007.

* Postal address: Faculty of Mathematics and Informatics, Vilnius University, Naugarduko 24, 03225 Vilnius, Lithuania.

Email address: remigijus.leipus@mif.vu.lt

** Postal address: Institute of Mathematics and Informatics, Akademijos 4, 08663 Vilnius, Lithuania. 
above results for the $\mathrm{G} / \mathrm{G} / 1 / 0$ queueing system (where customers arriving when the system is busy are rejected), in terms of interarrival and service length distributions.

The ON/OFF process (with independent or dependent periods $X^{\text {on }}$ and $Y^{\text {off }}$ ) is a particular case of a general class of regenerative processes or random tours; see e.g. Smith (1958). The probability distribution of a random tour starting at 0 is completely determined by the tour distribution $(\{y(t), t \geq 0\}, Z)$, where $Z$ is the length of tour (regeneration interval) and $\{y(t), t \geq 0\}$ is the tour process. In Lemma 2.1, below, we obtain a closed form of the covariance function of a general stationary random tour $\{X(t), t \geq 0\}$ in terms of the renewal function of regeneration points and the expectations $G^{0}(t)=\mathrm{E}\left[y(Z-t) \mathbf{1}_{\{t<Z\}}\right]$ (the 'backward tour mean'), $G^{1}(t)=\mathrm{E}\left[y_{(}(t) \mathbf{1}_{\{t<Z\}}\right]$ (the 'forward tour mean'), and $R(t)=$ $\mathrm{E}\left[y_{0}(0) \mathcal{y}_{0}(t) \mathbf{1}_{\left\{t<Z_{0}\right\}}\right]$, where $\left(\left\{\mathcal{y}_{0}(t), t \geq 0\right\}, Z_{0}\right)$ is the initial tour and $\mathbf{1}_{\{\cdot\}}$ is the indicator function. The expression for $\operatorname{cov}(X(0), X(t))$ in Lemma 2.1, below, is similar to the equation for the covariance of the ON/OFF process and can be analyzed by renewal methods developed by Heath et al. (1998). For the processes $A(t)$ and $W(t)$ discussed above, the asymptotic behaviour of the functions $G^{0}(t), G^{1}(t)$, and $R(t)$ as $t \rightarrow \infty$ is relatively easy to investigate; see Section 3. For regenerative processes, the asymptotic behaviour of $G^{0}(t), G^{1}(t)$, and $R(t)$ may be rather difficult to investigate; Lemma 2.1, below, can be regarded as a preliminary step in establishing the LRD property of such processes.

The LRD property (1.1) is also related to the concept of long-range count dependence (LRcD), introduced by Daley and Vesilo (1997). The LRcD property is defined in terms of asymptotic growth of the variance $\operatorname{var}(N(0, t])$ of the number of counts of a stationary point process in a (large) interval $(0, t]$. Daley and Vesilo (1997), (2000) obtained the LRcD property and the Hurst indices for several classes of point processes (not necessarily renewal) and queueing systems. Formally, a counting process is a particular case of the (integrated) regenerative process with tour $y(t)=\delta(t)$, where $\delta(\cdot)$ is the $\delta$-function. In Section 3 we obtain the asymptotics of the covariance function of counts of ON and OFF times in a finite moving interval $I+t$, from which the LRcD property in the renewal case easily follows.

\section{A stationary regenerative process and its covariance function}

Let $\{y(t), t \geq 0\}$ be a real-valued measurable stochastic process and let $Z>0$ be a random variable defined on the same probability space. Let

$$
\left(\left\{y_{j}(t), t \geq 0\right\}, Z_{j}\right), \quad j=1,2, \ldots,
$$

be i.i.d. copies of $(\{y(t), t \geq 0\}, Z)$. Let $\left(\left\{y_{0}(t), t \geq 0\right\}, Z_{0}\right)$ be independent of (2.1), generally having a different distribution. Define $T_{0}:=Z_{0}, T_{n}:=\sum_{i=1}^{n} Z_{i}, n \geq 1$, and consider a stationary regenerative process with regeneration points $T_{0}, T_{1}, \ldots$ defined by the following equality:

$$
X(t):=y_{0}(t) \mathbf{1}_{\left\{t<T_{0}\right\}}+\sum_{j=1}^{\infty} y_{j}\left(t-T_{j-1}\right) \mathbf{1}_{\left\{T_{j-1} \leq t<T_{j}\right\}}, \quad t \geq 0 .
$$

Assume that $\mu=\mathrm{E}[Z]<\infty$. Then, from Franken et al. (1981, Theorem 1.5.4), for any $p \geq 0$ we have

$$
\mathrm{E}\left[|X(t)|^{p}\right]=\mu^{-1} \int_{0}^{\infty} \mathrm{E}\left[|\mathcal{Y}(s)|^{p} \mathbf{1}_{\{Z>s\}}\right] \mathrm{d} s=\mu^{-1} \int_{0}^{\infty} \mathrm{E}\left[|\mathcal{Y}(Z-u)|^{p} \mathbf{1}_{\{Z>u\}}\right] \mathrm{d} u .
$$


Let $F(x):=\mathrm{P}[Z \leq x]$. Let $F^{\star n}$ denote the $n$th Stieltjes convolution of $F$ with itself (where $\left.F^{\star 0}(x):=\mathbf{1}_{\{x \geq 0\}}\right)$ and let

$$
U(t):=\mathrm{E}\left[N[0, t] \mid T_{0}=0\right]=\sum_{n=0}^{\infty} F^{\star n}(t)
$$

be the renewal function, where $N[0, t]:=\sum_{j=0}^{\infty} \mathbf{1}_{\left\{0 \leq T_{j} \leq t\right\}}$ is the number of renewal points in $[0, t]$ in a pure renewal process having a point at 0 . Next, for any $t>0$, define

$$
\begin{aligned}
z(t) & :=\int_{0}^{t} G^{0}(x) G^{1}(t-x) \mathrm{d} x, \\
R(t) & :=\mathrm{E}\left[X(0) X(t) \mathbf{1}_{\left\{t<T_{0}\right\}}\right]=\mathrm{E}\left[y_{0}(0) y_{0}(t) \mathbf{1}_{\left\{t<T_{0}\right\}}\right],
\end{aligned}
$$

where

$$
G^{0}(t):=\mathrm{E}\left[y(Z-t) \mathbf{1}_{\{t<Z\}}\right], \quad G^{1}(t):=\mathrm{E}\left[y(t) \mathbf{1}_{\{t<Z\}}\right] .
$$

In the sequel, we call $G^{0}(t)$ a backward tour mean and $G^{1}(t)$ a forward tour mean.

In the following lemma, we obtain the mean and covariance of the stationary regenerative process in (2.2).

Lemma 2.1. Assume that

$$
\mathrm{E}\left[X^{2}(0)\right]=\mu^{-1} \int_{0}^{\infty} \mathrm{E}\left[y^{2}(s) \mathbf{1}_{\{Z>s\}}\right] \mathrm{d} s<\infty .
$$

Then, for any $t \geq 0$, we have

$$
\begin{aligned}
\mathrm{E}[X(t)] & =\mu^{-1} \int_{0}^{\infty} G^{0}(s) \mathrm{d} s=\mu^{-1} \int_{0}^{\infty} G^{1}(s) \mathrm{d} s \\
\operatorname{cov}(X(0), X(t)) & =R(t)+\mu^{-1} \int_{0}^{t} z(t-s) U(\mathrm{~d} s)-\left(\frac{m}{\mu^{2}}\right)
\end{aligned}
$$

where

$$
m:=\int_{0}^{\infty} z(s) \mathrm{d} s=(\mu \mathrm{E}[X(0)])^{2} .
$$

Proof. Relations (2.7) and (2.3) imply that $\int_{0}^{\infty}\left|G^{i}(s)\right|^{p} \mathrm{~d} s<\infty, \quad p=1,2, i=0,1$; in particular,

$$
\int_{0}^{\infty}\left(G^{1}(s)\right)^{2} \mathrm{~d} s=\int_{0}^{\infty}\left(\mathrm{E}\left[y(s) \mathbf{1}_{\{s<Z\}}\right]\right)^{2} \mathrm{~d} s \leq \int_{0}^{\infty} \mathrm{E}\left[y^{2}(s) \mathbf{1}_{\{s<Z\}}\right] \mathrm{d} s=\mu \mathrm{E}\left[X^{2}(0)\right] .
$$

Therefore, the functions $z(t)$ and $R(t)$ in (2.4) and (2.5) respectively, are well-defined and bounded, i.e.

$$
|z(t)| \leq \mu \mathrm{E}\left[X^{2}(0)\right], \quad|R(t)| \leq \mathrm{E}\left[X^{2}(0)\right], \quad \text { for all } t \geq 0 .
$$


Relations (2.8) and (2.10) follow from (2.3) and the definitions in (2.4) and (2.6). Consider (2.9). Using (2.4), (2.5), (2.6), and the independence of disjoint tours, we obtain

$$
\begin{aligned}
\mathrm{E}[X(0) X(t)] & =\mathrm{E}\left[X(0) X(t) \mathbf{1}_{\left\{t<T_{0}\right\}}\right]+\sum_{i=0}^{\infty} \mathrm{E}\left[X(0) X(t) \mathbf{1}_{\left\{T_{i} \leq t<T_{i+1}\right\}}\right] \\
& =R(t)+\sum_{n=0}^{\infty} \int_{\left\{0<s_{0} \leq s_{1}<t\right\}} \mathrm{E}\left[\mathcal{Y}_{0}(0) \mathbf{1}_{\left\{T_{0} \in \mathrm{d} s_{0}\right\}} F^{n \star}\left(\mathrm{d} s_{1}-s_{0}\right) \mathcal{y}\left(t-s_{1}\right) \mathbf{1}_{\left\{s_{1}<t\right\}}\right] \\
& =R(t)+\mu^{-1} \int_{\left\{0<s_{0} \leq s_{1}<t\right\}} G^{0}\left(s_{0}\right) \mathrm{d} s_{0} U\left(\mathrm{~d} s_{1}-s_{0}\right) G^{1}\left(t-s_{1}\right) \\
& =R(t)+\mu^{-1} \int_{0}^{t} z(t-s) U(\mathrm{~d} s)
\end{aligned}
$$

which proves (2.9).

\section{The LRD property of stationary ON/OFF processes and forward recurrence time processes}

Let $\left(X^{\text {on }}, Y^{\text {off }}\right),\left(X_{1}, Y_{1}\right),\left(X_{2}, Y_{2}\right), \ldots$ be an i.i.d. sequence of random vectors taking values in $(0, \infty)^{2}$ and with arbitrarily dependent components, and let

$$
Z:=X^{\text {on }}+Y^{\text {off }}, \quad Z_{i}:=X_{i}+Y_{i}, \quad i \geq 1 .
$$

We shall assume that $\mu=\mathrm{E}[Z]=\mu_{\mathrm{on}}+\mu_{\mathrm{off}}<\infty$, where $\mu_{\mathrm{on}}:=\mathrm{E}\left[X^{\mathrm{on}}\right]$ and $\mu_{\mathrm{off}}:=\mathrm{E}\left[Y^{\mathrm{off}}\right]$. Consider a stationary version of the alternating point process

$$
0 \leq X_{0}^{\text {on }}<T_{0}<T_{0}+X_{1}<T_{1}<T_{1}+X_{2}<\cdots,
$$

where

$$
T_{0}:=X_{0}^{\mathrm{on}}+Y_{0}^{\mathrm{off}}, \quad T_{n}:=T_{0}+\sum_{i=1}^{n}\left(X_{i}+Y_{i}\right), \quad n \geq 1 .
$$

Intervals $\left[0, X_{0}^{\mathrm{on}}\right),\left[T_{i}, T_{i}+X_{i+1}\right), \quad i=0,1, \ldots$, will be called $O N$ intervals and intervals $\left[0=X_{0}^{\text {on }}, T_{0}\right),\left[T_{i}+X_{i+i}, T_{i+1}\right), i=0,1, \ldots$, will be called $O F F$ intervals. The initial time 0 falls into an ON or OFF interval depending on whether $X_{0}^{\text {on }}>0$ or $X_{0}^{\text {on }}=0$ respectively.

With the alternating point process in (3.2) we associate two stationary processes, the ON/OFF process $\{A(t), t \geq 0\}$ and the forward recurrence time of the busy period $\{W(t), t \geq 0\}$ as follows:

$$
\begin{aligned}
A(t) & :=\mathbf{1}_{\left\{X_{0}^{\mathrm{on}}>t\right\}}+\sum_{n=0}^{\infty} \mathbf{1}_{\left\{T_{n} \leq t<T_{n}+X_{n+1}\right\}}, \\
W(t) & :=\left(X_{0}^{\mathrm{on}}-t\right)_{+}+\sum_{n=0}^{\infty}\left(T_{n}+X_{n+1}-t\right)_{+} \mathbf{1}_{\left\{T_{n} \leq t<T_{n+1}\right\}},
\end{aligned}
$$

where $a_{+}=\max (a, 0)$. In the case of the G/G/1/0 queue, the process $W(t)$ represents the current workload in the system; see Section 4. In the sequel, we call $W(t)$ in (3.3) a workload process. It is clear from the definition that the processes $A(t)$ and $W(t)$ are regenerative in the 
sense of (2.2), with the tour duration $Z$ given in (3.1), and the corresponding tour processes given by

$$
y^{A}(t):=\mathbf{1}_{\left\{t<X^{\mathrm{on}}\right\}}, \quad y^{W}(t):=\left(X^{\mathrm{on}}-t\right)_{+} .
$$

By stationarity, we have $\mathrm{P}\left[X_{0}^{\mathrm{on}} \in \mathrm{d} x\right]=\mu^{-1} \mathrm{P}\left[X^{\text {on }}>x\right] \mathrm{d} x, x>0$, therefore

$$
\begin{aligned}
& \mathrm{E}[A(t)]=\mu^{-1} \int_{0}^{\infty} \mathrm{P}\left[X^{\text {on }}>s\right] \mathrm{d} s=\frac{\mu_{\mathrm{on}}}{\mu}, \\
& \mathrm{E}[W(t)]=\mu^{-1} \int_{0}^{\infty} \mathrm{E}\left[\left(X^{\text {on }}-s\right)_{+}\right] \mathrm{d} s=\frac{\mathrm{E}\left[\left(X^{\text {on }}\right)^{2}\right]}{2 \mu} .
\end{aligned}
$$

Moreover,

$$
\begin{aligned}
G_{A}^{0}(t) & =\mathrm{P}\left[Y^{\text {off }}<t \leq X^{\text {on }}+Y^{\text {off }}\right]=\overline{F_{\text {off }}}(t)-F(t), \\
G_{A}^{1}(t) & =\mathrm{P}\left[X^{\text {on }}>t\right]=\overline{F_{\text {on }}}(t), \\
R_{A}(t) & =\mathrm{P}\left[X_{0}^{\text {on }}>t\right], \\
G_{W}^{0}(t) & =\mathrm{E}\left[\left(t-Y^{\text {off }}\right)_{+} \mathbf{1}_{\left\{X^{\text {on }}+Y^{\text {off }}>t\right\}}\right], \\
G_{W}^{1}(t) & =\mathrm{E}\left[\left(X^{\text {on }}-t\right)_{+}\right], \\
R_{W}(t) & =\mu^{-1} \int_{t}^{\infty} x(x-t) \overline{F_{\text {on }}}(x) \mathrm{d} x .
\end{aligned}
$$

By definition and from (2.3), we have

$$
\int_{0}^{\infty} G_{W}^{0}(t) \mathrm{d} t=\int_{0}^{\infty} G_{W}^{1}(t) \mathrm{d} t=\mu \mathrm{E}[W(0)] .
$$

Next, for any $t>0$, define

$$
\begin{aligned}
z_{A}(t) & :=\int_{0}^{t} \overline{F_{\text {off }}}(x) \overline{F_{\text {on }}}(t-x) \mathrm{d} x, \\
z_{W}(t) & :=\int_{0}^{t} G_{W}^{0}(x) G_{W}^{1}(t-x) \mathrm{d} x .
\end{aligned}
$$

Corollary 3.1. (i) For any $t \geq 0$, we have

$$
\operatorname{cov}(A(0), A(t))=\frac{\mu_{\mathrm{off}} \mu_{\mathrm{on}}}{\mu^{2}}-\frac{1}{\mu} \int_{0}^{t} z_{A}(t-s) U(\mathrm{~d} s) .
$$

(ii) Assume in addition that $\mathrm{E}\left[\left(X^{\mathrm{on}}\right)^{3}\right]<\infty$. Then, for any $t \geq 0$, we have

$$
\operatorname{cov}(W(0), W(t))=R_{W}(t)+\frac{1}{\mu} \int_{0}^{t} z_{W}(t-s) U(\mathrm{~d} s)-(\mathrm{E}[W(0)])^{2} .
$$

Note that (3.8), the equation for the covariance of $A(t)$, is the same as in the case of independent ON and OFF periods; see Heath et al. (1998, Equation (2.7)). Using the results of Heath et al. (1998), we therefore obtain the following corollary. 
Corollary 3.2. Let

$$
\begin{aligned}
& \overline{F_{\text {on }}}(t)=t^{-\alpha_{\text {on }}} L_{\text {on }}(t), \\
& \overline{F_{\text {off }}}(t)=o\left(\overline{F_{\text {on }}}(t)\right), \quad t \rightarrow \infty,
\end{aligned}
$$

where $1<\alpha_{\mathrm{on}}<2$ and where $L_{\mathrm{on}}$ is a function, slowly varying at infinity. Assume, moreover, that there exists an $n \geq 1$ such that $F^{\star n}$ is nonsingular, where we recall that $F$ is the distribution function of $Z=X^{\mathrm{on}}+Y^{\mathrm{off}}$. Then we obtain

$$
\operatorname{cov}(A(0), A(t)) \sim \frac{\mu_{\mathrm{off}}^{2}}{\left(\alpha_{\mathrm{on}}-1\right) \mu^{3}} t^{-\left(\alpha_{\mathrm{on}}-1\right)} L_{\mathrm{on}}(t), \quad t \rightarrow \infty .
$$

Proof. Note that (3.9) and (3.10) imply

$$
\begin{aligned}
\bar{F}(t) & \sim L_{\mathrm{on}}(t) t^{-\alpha_{\mathrm{on}}}, \\
z_{A}(t) & \sim \mu_{\mathrm{off}} L_{\mathrm{on}}(t) t^{-\alpha_{\mathrm{on}}},
\end{aligned}
$$

where (3.12) is proved in Lemma 3.1, below. Relation (3.13) follows from (3.12), (3.9), (3.10), and Heath et al. (1998, Lemma 4.2). Finally, (3.11) follows from (3.8), (3.13), and the argument in Heath et al. (1998, proof of Theorem 4.3).

Consider now the LRcD property of the introduced ON/OFF process. Let $I \subset(0, \infty)$ be a (fixed) interval of a finite Lebesgue measure $|I|$. Introduce the counting processes

$$
\begin{aligned}
& N_{\text {on }}(I)=\sum_{n=0}^{\infty} \mathbf{1}_{\left\{T_{n} \in I\right\}}, \\
& N_{\text {off }}(I)=\mathbf{1}_{\left\{X_{0}^{\mathrm{on}} \in I\right\}}+\sum_{n=0}^{\infty} \mathbf{1}_{\left\{T_{n}+X_{n+1} \in I\right\}} .
\end{aligned}
$$

Note that the processes $N_{\mathrm{on}}(I+t)$ and $N_{\mathrm{off}}(I+t), t \geq 0$, are both stationary and

$$
\begin{aligned}
\mathrm{E}\left[N_{\text {on }}(I)\right] & =\mathrm{E}\left[N_{\text {off }}(I)\right] \\
& =\frac{|I|}{\mu}, \\
\operatorname{cov}\left(N_{\text {on }}(I), N_{\text {on }}(I+t)\right) & =\int_{I} \int_{I+t} \mathrm{P}\left[N_{\text {on }}(\mathrm{d} x)=1, N_{\text {on }}(\mathrm{d} y)=1\right]-\left(\frac{|I|}{\mu}\right)^{2} \\
& =\mu^{-1} \int_{I}\left(U(I+t-x)-\mu^{-1}|I|\right) \mathrm{d} x, \\
\operatorname{cov}\left(N_{\text {off }}(I), N_{\text {off }}(I+t)\right) & =\int_{I} \int_{I+t} \mathrm{P}\left[N_{\text {off }}(\mathrm{d} x)=1, N_{\text {off }}(\mathrm{d} y)=1\right]-\left(\frac{|I|}{\mu}\right)^{2} \\
& =\mu^{-1} \int_{I}\left(\left(F_{\text {off }} \star U \star F_{\text {on }}\right)(I+t-x)-\mu^{-1}|I|\right) \mathrm{d} x,
\end{aligned}
$$

provided that $I \cap(I+t)=\varnothing$. 
Corollary 3.3. Assume the same conditions as in Corollary 3.2. Then, as $t \rightarrow \infty$, we obtain

$$
\begin{aligned}
& \left.\begin{array}{c}
\operatorname{cov}\left(N_{\text {on }}(I), N_{\text {on }}(I+t)\right) \\
\operatorname{cov}\left(N_{\text {off }}(I), N_{\text {off }}(I+t)\right)
\end{array}\right\} \sim \frac{|I|^{2}}{\left(\alpha_{\text {on }}-1\right) \mu^{3}} t^{-\left(\alpha_{\text {on }}-1\right)} L_{\text {on }}(t), \\
& \left.\begin{array}{r}
\operatorname{var}\left(N_{\text {on }}[0, t]\right) \\
\operatorname{var}\left(N_{\text {off }}[0, t]\right)
\end{array}\right\} \sim \frac{2}{\left(\alpha_{\text {on }}-1\right)\left(2-\alpha_{\text {on }}\right)\left(3-\alpha_{\text {on }}\right) \mu^{3}} t^{3-\alpha_{\text {on }}} L_{\text {on }}(t) .
\end{aligned}
$$

Proof. Let $\overline{F_{2}}(x):=\mu^{-1} \int_{x}^{\infty} \bar{F}(y) \mathrm{d} y$. From Heath et al. (1998, Equation (3.12)) and Lemma 3.1, below,

$$
U(I+t)-\mu^{-1}|I| \sim \mu^{-1} \int_{I+t} \overline{F_{2}}(x) \mathrm{d} x \sim \frac{|I|}{\left(\alpha_{\mathrm{on}}-1\right) \mu^{2}} t^{-\left(\alpha_{\mathrm{on}}-1\right)} L_{\mathrm{on}}(t),
$$

implying the first asymptotic relation in (3.16) by (3.14) and by the dominated convergence theorem. The second relation in (3.16) follows analogously from (3.15) and

$$
\left(F_{\text {off }} \star U \star F_{\text {on }}\right)(I+t)-\mu^{-1}|I| \sim \frac{|I|}{\left(\alpha_{\text {on }}-1\right) \mu^{2}} t^{-\left(\alpha_{\text {on }}-1\right)} L_{\text {on }}(t) .
$$

Relation (3.19) follows from Heath et al. (1998, Theorem 3.1(ii)), the fact that $\int_{0}^{\infty} \bar{F}(y) \mathrm{d} y=$ $\int_{0}^{\infty} \overline{F_{\text {on }} \star F_{\text {off }}}(y) \mathrm{d} y=\mu$, and (3.18). Relation (3.17) is immediate from (3.16) and $\alpha_{\text {on }} \in$ $(1,2)$.

Remark 3.1. Note that the covariance $\operatorname{cov}(A(0), A(t))=\operatorname{cov}(1-A(0), 1-A(t))$ as well as the covariances in (3.14) and (3.15) are symmetric with respect to the change of $F_{\text {on }}$ by $F_{\text {off }}$ and vice versa. Accordingly, Corollaries 3.2 and 3.3 remain valid, with obvious changes, if conditions on $\mathrm{ON}$ and OFF intervals are exchanged.

Lemma 3.1. Let $X, Y \geq 0$ be arbitrarily dependent random variables such that

(i) $\mathrm{P}[X>t]=L(t) t^{-\gamma}$,

(ii) $\mathrm{P}[Y>t]=o(\mathrm{P}[X>t])$ as $t \rightarrow \infty$,

where $\gamma>0$ and $L$ is slowly varying at infinity. Let $Z:=X+Y$, then

$$
\mathrm{P}[Z>t] \sim L(t) t^{-\gamma} .
$$

Proof. Let $0<\varepsilon<1$. Then we have

$$
\begin{aligned}
\mathrm{P}[X>t] & \leq \mathrm{P}[X+Y>t] \\
& \leq \mathrm{P}[Y>\varepsilon t]+\mathrm{P}[Y \leq \varepsilon t, X+Y>t] \\
& \leq \mathrm{P}[Y>\varepsilon t]+\mathrm{P}[X>(1-\varepsilon) t] .
\end{aligned}
$$

Dividing the above inequality by $\mathrm{P}[X>t]$, we obtain

$$
1 \leq \liminf _{t \rightarrow \infty} \frac{\mathrm{P}[Z>t]}{L(t) t^{-\gamma}} \leq \limsup _{t \rightarrow \infty} \frac{\mathrm{P}[Z>t]}{L(t) t^{-\gamma}} \leq(1-\varepsilon)^{-\gamma},
$$

where we used the following fact:

$$
\limsup _{t \rightarrow \infty} \frac{\mathrm{P}[Y>\varepsilon t]}{L(t) t^{-\gamma}} \leq \limsup _{t \rightarrow \infty} \frac{\mathrm{P}[Y>\varepsilon t]}{\mathrm{P}[X>\varepsilon t]} \limsup _{t \rightarrow \infty} \frac{L(\varepsilon t)(\varepsilon t)^{-\gamma}}{L(t) t^{-\gamma}}=0
$$


(by assumptions (i) and (ii)), while lim $\sup _{t \rightarrow \infty} L((1-\varepsilon) t)((1-\varepsilon) t)^{-\gamma} / L(t) t^{-\gamma}=(1-\varepsilon)^{-\gamma}$. As $\varepsilon>0$ is arbitrary in (3.20), this completes the proof.

Next, we turn to the asymptotics of $\operatorname{cov}(W(0), W(t))$. According to Corollary 3.1, this can be written as

$$
\operatorname{cov}(W(0), W(t)))=R_{W}(t)-\mu^{-1} h(t),
$$

where the function $R_{W}(t)$ is defined in (3.6) and $h(t):=(m / \mu)-\int_{0}^{t} z_{W}(t-x) U(\mathrm{~d} x)$, with $z_{W}(t)$ given in (3.7). The next corollary encompasses the following two cases:

(i) $h(t)=o\left(R_{W}(t)\right)$,

(ii) $R_{W}(t)=o(h(t))$.

In case (i), the asymptotics of $\operatorname{cov}(W(0), W(t))$ coincides with that of $R_{W}(t)$, and in case (ii), it coincides with the asymptotics of $-\mu^{-1} h(t)$.

Corollary 3.4. Assume that the following conditions hold: $\mathrm{E}\left[\left(X^{\mathrm{on}}\right)^{3}\right]<\infty, F^{\star n}$ is nonsingular for some $n \geq 1$, and

$$
\begin{aligned}
& \overline{F_{\mathrm{off}}}(t)=t^{-\alpha_{\mathrm{off}}} L_{\mathrm{off}}(t), \\
& G_{W}^{0}(t)=o\left(\overline{F_{\mathrm{off}}}(t)\right), \quad t \rightarrow \infty,
\end{aligned}
$$

where $1<\alpha_{\mathrm{off}}<2$ and $L_{\mathrm{off}}$ is slowly varying at infinity.

(i) Let

$$
\overline{F_{\mathrm{on}}}(t)=t^{-\alpha_{\mathrm{on}}} L_{\mathrm{on}}(t),
$$

where $3<\alpha_{\mathrm{on}}<\alpha_{\mathrm{off}}+2$ and $L_{\mathrm{on}}$ is slowly varying at infinity. Then

$$
\operatorname{cov}(W(0), W(t)) \sim R_{W}(t) \sim \frac{1}{\mu\left(\alpha_{\mathrm{on}}-3\right)\left(\alpha_{\mathrm{on}}-2\right)} t^{-\left(\alpha_{\mathrm{on}}-3\right)} L_{\mathrm{on}}(t) .
$$

(ii) Let

$$
\overline{F_{\text {on }}}(t)=o\left(t^{-2} \overline{F_{\text {off }}}(t)\right)
$$

Then

$$
\operatorname{cov}(W(0), W(t)) \sim-\mu^{-1} h(t) \sim \frac{m}{\left(\alpha_{\mathrm{off}}-1\right) \mu^{3}} t^{-\left(\alpha_{\mathrm{off}}-1\right)} L_{\mathrm{off}}(t) .
$$

Proof. (i) Note that (3.24) implies

$$
R_{W}(t)=\frac{1}{\mu} \int_{t}^{\infty} x(x-t) x^{-\alpha_{\mathrm{on}}} L_{\mathrm{on}}(x) \mathrm{d} x \sim \frac{1}{\mu\left(\alpha_{\mathrm{on}}-3\right)\left(\alpha_{\mathrm{on}}-2\right)} t^{-\left(\alpha_{\mathrm{on}}-3\right)} L_{\mathrm{on}}(t) .
$$

Hence, by (3.21), (3.25) reduces to

$$
h(t)=o\left(t^{-\left(\alpha_{\mathrm{on}}-3\right)} L_{\mathrm{on}}(t)\right) .
$$

Let us prove that $\mathrm{E}\left[\left(X^{\mathrm{on}}\right)^{3}\right]<\infty$ and that (3.22) and (3.23) (where $1<\alpha_{\text {off }}<2$ ) imply

$$
z_{W}(t)=o(\bar{F}(t))=o\left(L_{\mathrm{off}}(t) t^{-\alpha_{\mathrm{off}}}\right), \quad t \rightarrow \infty .
$$


Indeed, by (3.5) and the Chebyshev inequality, we have $G_{W}^{1}(t) \leq t^{-2} \mathrm{E}\left[\left(X^{\mathrm{on}}\right)^{3}\right]$. Using this fact and (3.23), we obtain

$$
\begin{aligned}
z_{W}(t) & =\int_{0}^{t / 2} G_{W}^{0}(x) G_{W}^{1}(t-x) \mathrm{d} x+\int_{t / 2}^{t} G_{W}^{0}(x) G_{W}^{1}(t-x) \mathrm{d} x \\
& \leq C\left(\frac{t}{2}\right)^{-2} \int_{0}^{\infty} G_{W}^{0}(x) \mathrm{d} x+o\left(L_{\mathrm{off}}(t) t^{-\alpha_{\mathrm{off}}}\right) \sup _{t / 2 \leq x \leq t} \frac{L_{\mathrm{off}}(x) x^{-\alpha_{\mathrm{off}}}}{L_{\mathrm{off}}(t) t^{-\alpha_{\mathrm{off}}}} \int_{0}^{\infty} G_{W}^{1}(x) \mathrm{d} x \\
& =O\left(t^{-2}\right)+o\left(L_{\mathrm{off}}(t) t^{-\alpha_{\mathrm{off}}}\right) \\
& =o\left(L_{\mathrm{off}}(t) t^{-\alpha_{\mathrm{off}}}\right)
\end{aligned}
$$

since $\alpha_{\text {off }}<2$. This proves (3.29). Using (3.29) and Heath et al. (1998, Theorem 3.1(iii)), we obtain

$$
h(t) \sim-\frac{m}{\left(\alpha_{\text {off }}-1\right) \mu^{2}} t^{-\left(\alpha_{\text {off }}-1\right)} L_{\text {off }}(t), \quad t \rightarrow \infty,
$$

which implies (3.28), since $\alpha_{\text {on }}<\alpha_{\text {off }}+2$.

(ii) Equation (3.27) follows from (3.21), (3.30), and $R_{W}(t)=o(h(t))$; in other words, it follows from

$$
R_{W}(t)=\mu^{-1} \int_{t}^{\infty} x(x-t) \overline{F_{\text {on }}}(x) \mathrm{d} x=o\left(t^{-\left(\alpha_{\text {off }}-1\right)} L_{\text {off }}(t)\right)
$$

The last fact follows from (3.26), (3.22), and $\int_{t}^{\infty} x(x-t) x^{-2} \overline{F_{\mathrm{off}}}(x) \mathrm{d} x=O\left(t^{1-\alpha_{\mathrm{off}}} L_{\mathrm{off}}(t)\right)$, which completes the proof.

Corollary 3.5. Assume that the following conditions hold: $\overline{F_{\mathrm{on}}}(t)=t^{-\alpha_{\mathrm{on}}} L_{\mathrm{on}}(t), \overline{F_{\mathrm{off}}}(t)=$ $t^{-\alpha_{\mathrm{off}}} L_{\mathrm{off}}(t), F^{n \star}$ is nonsingular for some $n \geq 1$, and (3.23), where $3<\alpha_{\mathrm{on}}<4,1<\alpha_{\mathrm{off}}<2$, and $\alpha_{\mathrm{on}} \neq \alpha_{\mathrm{off}}+2$. Then, $W(t)$ in (3.3) has the LRD property with rate $\min \left(\alpha_{\mathrm{on}}-3, \alpha_{\mathrm{off}}-1\right) \in$ $(0,1)$.

Note that all the conditions of Corollaries 3.2-3.5, with the exception of (3.23) and the obviously mild condition that $F^{\star n}$ is nonsingular for some $n \geq 0$, are formulated in terms of marginal distributions of $\mathrm{ON}$ and OFF periods. We show below that assumption (3.23) is also not very restrictive, in the sense that it holds in the case of independent ON and OFF periods.

Proposition 3.1. Assume that $X^{\mathrm{on}}$ and $Y^{\mathrm{off}}$ are independent, $\mathrm{E}\left[\left(X^{\mathrm{on}}\right)^{3}\right]<\infty$, and (3.22) is satisfied, with $1<\alpha_{\text {off }}<2$. Then (3.23) holds.

Proof. Decompose $G_{W}^{0}(t)=J_{1}(t)+J_{2}(t)$, where

$$
J_{1}(t):=\int_{0}^{t(1-\varepsilon)}(t-u) \overline{F_{\mathrm{on}}}(t-u) F_{\mathrm{off}}(\mathrm{d} u), \quad J_{2}(t):=\int_{t(1-\varepsilon)}^{t}(t-u) \overline{F_{\mathrm{on}}}(t-u) F_{\mathrm{off}}(\mathrm{d} u) .
$$

Using $t \overline{F_{\text {on }}}(t) \leq C t^{-2}$, for all $t>0$, we obtain, for each $\varepsilon>0$,

$$
J_{1}(t) \leq C \int_{0}^{t(1-\varepsilon)}(t-u)^{-2} F_{\text {off }}(\mathrm{d} u) \leq C(t \varepsilon)^{-2}
$$


where the constant $C$ does not depend on $t$ and $\varepsilon$. On the other hand, using (3.22), we have

$$
\begin{aligned}
J_{2}(t) & \leq C \int_{t(1-\varepsilon)}^{t} F_{\mathrm{off}}(\mathrm{d} u) \\
& =C \frac{L_{\mathrm{off}}(t)}{(t(1-\varepsilon))^{\alpha_{\mathrm{off}}}}\left(\frac{L_{\mathrm{off}}(t(1-\varepsilon))}{\left.L_{\mathrm{off}}(t)\right)}-1\right)+C L_{\mathrm{off}}(t)\left(\frac{1}{(t(1-\varepsilon))^{\alpha_{\mathrm{off}}}}-\frac{1}{t^{\alpha_{\mathrm{off}}}}\right) \\
& =: J_{3}(t)+J_{4}(t) .
\end{aligned}
$$

Here, the term $J_{4}(t)=O\left(\varepsilon L_{\text {off }}(t) t^{-\alpha_{\text {off }}}\right)$; in other words, the ratio $J_{4}(t) /\left(L_{\text {off }}(t) t^{-\alpha_{\text {off }}}\right)$ can be made arbitrarily small uniformly in $t \geq 1$ by taking $\varepsilon>0$ to be small enough. Clearly, $J_{i}(t)=o\left(L_{\mathrm{off}}(t) t^{-\alpha_{\mathrm{off}}}\right), \quad i=1,3$, for any $\varepsilon>0$ fixed. This proves (3.23).

\section{Application to the $G / G / 1 / 0$ queue}

Consider a G/G/1/0 queueing system, with general interarrival distribution $F_{\tau}(x)=$ $\mathrm{P}[\tau \leq x]$ and general service distribution $F_{\sigma}(x)=\mathrm{P}[\sigma \leq x], \quad x \in(0, \infty)$. We assume that $\mu_{\sigma}:=\mathrm{E}[\sigma]<\infty$ and $\mu_{\tau}:=\mathrm{E}[\tau]<\infty$. The interarrival and service times $\tau_{i}, \sigma_{i}$, $i=1,2, \ldots$, are all independent, and customers arriving when the system is busy are rejected. The number of customers in the system at time $t, A(t)$, is equal to 1 or 0 . As noted in Section 1 , $A(t)$ is a particular case of the ON/OFF process with generally dependent $\mathrm{ON}$ and OFF intervals whose joint distribution is given by

$$
\begin{aligned}
\mathrm{P}\left[X^{\mathrm{on}} \in \mathrm{d} x, Y^{\mathrm{off}} \in \mathrm{d} y\right] & :=\sum_{i=1}^{\infty} \mathrm{P}\left[\sigma_{1} \in \mathrm{d} x, \tau_{1}+\cdots+\tau_{i-1} \leq x, \tau_{1}+\cdots+\tau_{i} \in \mathrm{d} y+x\right] \\
& =\mathrm{P}[\sigma \in \mathrm{d} x] \int_{0}^{x} \sum_{i=1}^{\infty} \mathrm{P}\left[\tau_{1}+\cdots+\tau_{i-1} \in \mathrm{d} u, \tau_{i} \in \mathrm{d} y+x-u\right] \\
& =F_{\sigma}(\mathrm{d} x) \int_{0}^{x} H(\mathrm{~d} u) F_{\tau}(x-u+\mathrm{d} y)
\end{aligned}
$$

where

$$
H(t):=\sum_{n=0}^{\infty} F_{\tau}^{\star n}(t)
$$

is the renewal function of the arrival process. A stationary version of the corresponding $\mathrm{G} / \mathrm{G} / \mathrm{1} / 0$ queue and the processes $A(t), W(t), t \geq 0$, can be constructed as in Borovkov (1976, Chapter 7) or Baccelli and Brémaud (1994, Chapter 2.5).

We obtain the following results about the asymptotics of the covariances of the processes $A(t)$ and $W(t)$ in the $\mathrm{G} / \mathrm{G} / 1 / 0$ queue with heavy tailed arrival and/or service times.

Proposition 4.1. Assume that $F^{\star n}$ is nonsingular for some $n \geq 1$, where $F(x)=\mathrm{P}\left[X^{\mathrm{on}}+\right.$ $\left.Y^{\text {off }} \leq x\right]$.

(i) Let $\overline{F_{\tau}}(x)=L_{\tau}(x) x^{-\alpha}$ and $\overline{F_{\sigma}}(x)=o\left(\overline{F_{\tau}}(x)\right)$, where $L_{\tau}$ is slowly varying at infinity and $1<\alpha<2$. Then, as $t \rightarrow \infty$, we have

$$
\operatorname{cov}(A(0), A(t)) \sim \frac{\mu_{\sigma}^{2}}{(\alpha-1) \mu^{2} \mu_{\tau}} t^{-(\alpha-1)} L_{\tau}(t),
$$

where $\mu:=\mu_{\sigma}+\mu_{\mathrm{off}}$ and $\mu_{\mathrm{off}}$ is given in (4.5). 
(ii) Let $\overline{F_{\sigma}}(x)=L_{\sigma}(x) x^{-\beta}$ and $\overline{F_{\tau}}(x)=o\left(\overline{F_{\sigma}}(x)\right)$, where $L_{\sigma}$ is slowly varying at infinity and $1<\beta<2$. Then, as $t \rightarrow \infty$, we have

$$
\operatorname{cov}(A(0), A(t)) \sim \frac{\left(\mu-\mu_{\sigma}\right)^{2}}{(\beta-1) \mu^{3}} t^{-(\beta-1)} L_{\sigma}(t) .
$$

Proposition 4.1 is a direct consequence of Corollary 3.2 and Lemma 4.1, below. Similarly, Corollary 3.4 and Lemmas 4.1-4.2, below, yield the following result.

Proposition 4.2. Assume that the following conditions hold: $\mathrm{E}\left[\sigma^{3}\right]<\infty, F^{\star n}$ is nonsingular for some $n \geq 1$, and $\overline{F_{\tau}}(x)=x^{-\alpha} L_{\tau}(x)$, where $1<\alpha<2$ and $L_{\tau}$ is slowly varying at infinity.

(i) Let $\overline{F_{\sigma}}(x)=x^{-\beta} L_{\sigma}(x)$, where $3<\beta<\alpha+2$ and $L_{\sigma}$ is slowly varying at infinity. Then

$$
\operatorname{cov}(W(0), W(t)) \sim \frac{1}{\mu(\beta-3)(\beta-2)} t^{-(\beta-3)} L_{\sigma}(t) .
$$

(ii) Let $\overline{F_{\sigma}}(x)=o\left(x^{-2} \overline{F_{\tau}}(x)\right)$. Then

$$
\operatorname{cov}(W(0), W(t)) \sim \frac{m}{(\alpha-1) \mu^{3}} t^{-(\alpha-1)} L_{\tau}(t),
$$

where $m:=\left(\mathrm{E}\left[\sigma^{2}\right]\right)^{2} / 4$ and $\mu$ is the same as in Proposition 4.1.

The following lemma is a straightforward consequence of (4.1).

Lemma 4.1. We have

$$
\begin{aligned}
\overline{F_{\mathrm{on}}}(x) & =\overline{F_{\sigma}}(x), \\
\mu_{\mathrm{on}} & =\mu_{\sigma} \\
\overline{F_{\mathrm{off}}}(x) & =\int_{0}^{\infty} H(\mathrm{~d} u) \int_{u}^{\infty} F_{\sigma}(\mathrm{d} w) \overline{F_{\tau}}(w-u+x), \\
\mu_{\mathrm{off}} & =\mu_{\tau} \int_{0}^{\infty} \overline{F_{\sigma}}(u) H(\mathrm{~d} u)-\mu_{\sigma}, \\
\overline{F_{\mathrm{off}}}(x) & \leq q \overline{F_{\tau}}(x)
\end{aligned}
$$

where $q:=\int_{0}^{\infty} \overline{F_{\sigma}}(u) H(\mathrm{~d} u)=\left(\mu_{\sigma}+\mu_{\mathrm{off}}\right) / \mu_{\tau}$. If, in addition, $F_{\tau}$ is long-tailed, i.e. for every $z \geq 0$

$$
\lim _{x \rightarrow \infty} \frac{\overline{F_{\tau}}(x+z)}{\overline{F_{\tau}}(x)}=1,
$$

then

$$
\overline{F_{\text {off }}}(x) \sim q \overline{F_{\tau}}(x), \quad x \rightarrow \infty .
$$

Proof. Note that the renewal equation $H(x)=1+\int_{0}^{t} F_{\tau}(x-u) H(\mathrm{~d} u)$ implies that, for every $x \geq 0$,

$$
\int_{0}^{x} \overline{F_{\tau}}(x-u) H(\mathrm{~d} u)=1, \quad \int_{0}^{x}\left(\int_{0}^{x-u} \overline{F_{\tau}}(z) \mathrm{d} z\right) H(\mathrm{~d} u)=x .
$$


Relations (4.2), (4.3), and (4.4) are immediate by (4.1) and (4.9). Consider (4.5). Using (4.4) and (4.1), we have

$$
\begin{aligned}
\mu_{\mathrm{off}} & =\int_{0}^{\infty} H(\mathrm{~d} u) \int_{u}^{\infty} F_{\sigma}(\mathrm{d} w) \int_{w-u}^{\infty} \overline{F_{\tau}}(z) \mathrm{d} z \\
& =\mu_{\tau} \int_{0}^{\infty} F_{\sigma}(\mathrm{d} w) \int_{0}^{w} H(\mathrm{~d} u)-\int_{0}^{\infty} F_{\sigma}(\mathrm{d} w) \int_{0}^{w} H(\mathrm{~d} u) \int_{0}^{w-u} \overline{F_{\tau}}(z) \mathrm{d} z .
\end{aligned}
$$

From the second equality in (4.9), (4.5) therefore follows.

Next, using (4.4), (4.5), (4.7), and the dominated convergence theorem, we obtain

$$
\begin{aligned}
\overline{F_{\text {off }}}(x) & =\overline{F_{\tau}}(x) \int_{0}^{\infty}\left\{\int_{0}^{\infty} \frac{\overline{F_{\tau}}(x+z)}{\overline{F_{\tau}}(x)} F_{\sigma}(u+\mathrm{d} z)\right\} H(\mathrm{~d} u) \\
& \sim \overline{F_{\tau}}(x) \int_{0}^{\infty} \overline{F_{\sigma}}(u) H(\mathrm{~d} u) \\
& =q \overline{F_{\tau}}(x)
\end{aligned}
$$

which proves (4.8). Relation (4.6) is immediate from (4.10) and $\overline{F_{\tau}}(x+z) \leq \overline{F_{\tau}}(x), x, z \geq 0$, and does not require (4.7). Therefore, the proof is complete.

Lemma 4.2. Assume that $\mathrm{E}\left[\sigma^{3}\right]<\infty$ and $\overline{F_{\tau}}(x)=L(x) x^{-\alpha}$, where $1<\alpha<2$ and $L$ is slowly varying at infinity. Let $G_{W}^{0}(t)$ be defined as in (3.4), from the joint distribution in (4.1). Then $G_{W}^{0}(t)=o\left(\overline{F_{\tau}}(t)\right)$.

Proof. From (4.1), we have

$$
\begin{aligned}
G_{W}^{0}(t) & =\int_{0}^{t}(t-u) \int_{t-u}^{\infty} \mathrm{P}\left[X^{\mathrm{on}} \in \mathrm{d} x, Y^{\mathrm{off}} \in \mathrm{d} u\right] \\
& =\int_{0}^{t}(t-u) \int_{t-u}^{\infty} F_{\sigma}(\mathrm{d} w) \int_{0}^{w} H(\mathrm{~d} z) F_{\tau}(w-z+\mathrm{d} u) \\
& =Q_{1}(t)+Q_{2}(t)
\end{aligned}
$$

where

$$
\begin{aligned}
Q_{1}(t) & :=\int_{0}^{t} F_{\sigma}(\mathrm{d} w) \int_{0}^{w} H(\mathrm{~d} z) \int_{0}^{w} x F_{\tau}(w-z+t-\mathrm{d} x), \\
Q_{2}(t) & :=\int_{t}^{\infty} F_{\sigma}(\mathrm{d} w) \int_{0}^{w} H(\mathrm{~d} z) \int_{0}^{t} x F_{\tau}(w-z+t-\mathrm{d} x) .
\end{aligned}
$$

The second term can be easily evaluated as follows using integration by parts, (4.9), and the Chebyshev inequality:

$$
\begin{aligned}
Q_{2}(t) & =\int_{t}^{\infty} F_{\sigma}(\mathrm{d} w) \int_{0}^{w} H(\mathrm{~d} z)\left(t \overline{F_{\tau}}(w-z)-\int_{0}^{t} \overline{F_{\tau}}(w-z+t-x) \mathrm{d} x\right) \\
& \leq t \int_{t}^{\infty} F_{\sigma}(\mathrm{d} w) \int_{0}^{w} \overline{F_{\tau}}(w-z) H(\mathrm{~d} z) \\
& =t \overline{F_{\sigma}}(t) \\
& \leq t^{-2} \mathrm{E}\left[\sigma^{3}\right] \\
& =o\left(L(t) t^{-\alpha}\right) .
\end{aligned}
$$


To evaluate $Q_{1}(t)$, we split it up, i.e.

$$
Q_{1}(t)=\int_{0}^{t_{*}} F_{\sigma}(\mathrm{d} w) \cdots+\int_{t_{*}}^{t} F_{\sigma}(\mathrm{d} w) \cdots=: Q_{11}(t)+Q_{12}(t),
$$

where $t_{*}=t^{\gamma}$ with $(1+\alpha) / 3<\gamma<1$. Similarly, as above, using integration by parts and (4.9), we have

$$
\begin{aligned}
Q_{12}(t) & =\int_{t_{*}}^{t} F_{\sigma}(\mathrm{d} w) \int_{0}^{w} H(\mathrm{~d} z)\left(w \overline{F_{\tau}}(t-z)-\int_{0}^{w} \overline{F_{\tau}}(w-z+t-x) \mathrm{d} x\right) \\
& \leq \int_{t_{*}}^{t} w F_{\sigma}(\mathrm{d} w) \int_{0}^{w} \overline{F_{\tau}}(t-z) H(\mathrm{~d} z) \\
& \leq \int_{t_{*}}^{t} w F_{\sigma}(\mathrm{d} w) \int_{0}^{w} \overline{F_{\tau}}(w-z) H(\mathrm{~d} z) \\
& =\int_{t_{*}}^{t} w F_{\sigma}(\mathrm{d} w) \\
& \leq t t_{*}^{-3} \mathrm{E}\left[\sigma^{3}\right] \\
& =o\left(L(t) t^{-\alpha}\right) .
\end{aligned}
$$

Consider the main term, $Q_{11}(t)$. Integrating by parts, we have

$$
\begin{aligned}
Q_{11}(t) & =\int_{0}^{t_{*}} F_{\sigma}(\mathrm{d} w) \int_{0}^{w} H(\mathrm{~d} z) \int_{0}^{w} x F_{\tau}(w-z+t-\mathrm{d} x) \\
& =\int_{0}^{t_{*}} F_{\sigma}(\mathrm{d} w) \int_{0}^{w} H(\mathrm{~d} z)\left(w \overline{F_{\tau}}(t-z)-\int_{0}^{w} \overline{F_{\tau}}(w-z+t-x) \mathrm{d} x\right) \\
& =P_{1}(t)-P_{2}(t),
\end{aligned}
$$

where

$$
\begin{aligned}
P_{1}(t) & :=\int_{0}^{t_{*}} w F_{\sigma}(\mathrm{d} w) \int_{0}^{w} H(\mathrm{~d} z)\left(\overline{F_{\tau}}(t-z)-\overline{F_{\tau}}(t)\right) \\
P_{2}(t) & :=\int_{0}^{t_{*}} F_{\sigma}(\mathrm{d} w) \int_{0}^{w} H(\mathrm{~d} z) \int_{0}^{w}\left(\overline{F_{\tau}}(w-z+t-x)-\overline{F_{\tau}}(t)\right) \mathrm{d} x .
\end{aligned}
$$

In view of (4.11) and (4.12), it remains to show that $P_{i}(t)=o\left(L(t) t^{-\alpha}\right), i=1,2$. We show that this relation holds for $P_{2}(t)$; the proof for $P_{1}(t)$ is analogous. To that end, write

$$
P_{2}(t)=\overline{F_{\tau}}(t)\left(p^{\prime}(t)+p^{\prime \prime}(t)\right),
$$

where

$$
\begin{aligned}
p^{\prime}(t) & :=\int_{0}^{t_{*}} F_{\sigma}(\mathrm{d} w) \int_{0}^{w} H(\mathrm{~d} z) \int_{0}^{w}\left(\frac{w-z+t-x}{t}\right)^{-\alpha}\left(\frac{L(w-z+t-x)}{L(t)}-1\right) \mathrm{d} x, \\
p^{\prime \prime}(t) & :=\int_{0}^{t_{*}} F_{\sigma}(\mathrm{d} w) \int_{0}^{w} H(\mathrm{~d} z) \int_{0}^{w}\left(\left(1+\frac{w-z-x}{t}\right)^{-\alpha}-1\right) \mathrm{d} x .
\end{aligned}
$$


Using the dominated convergence theorem, a well-known property of slowly varying functions, and $H(w)=O(w)$, we see that

$$
\begin{aligned}
p^{\prime}(t) & =O\left(\delta_{t} \int_{0}^{t_{*}} F_{\sigma}(\mathrm{d} w) \int_{0}^{w} w H(\mathrm{~d} z)\right) \\
& =O\left(\delta_{t} \int_{0}^{\infty} w H(w) F_{\sigma}(\mathrm{d} w)\right) \\
& =O\left(\delta_{t}\right) \\
& =o(1)
\end{aligned}
$$

where $\delta_{t} \rightarrow 0$ as $t \rightarrow 0$. Finally,

$$
\begin{aligned}
\left|p^{\prime \prime}(t)\right| & \leq C t^{-1} \int_{0}^{t_{*}} F_{\sigma}(\mathrm{d} w) \int_{0}^{w} H(\mathrm{~d} z) \int_{0}^{w}(w-z-x) \mathrm{d} x \\
& \leq C t^{-1} \int_{0}^{t_{*}} w H(w) F_{\sigma}(\mathrm{d} w) \\
& =O\left(t^{-1}\right) \\
& =o(1) .
\end{aligned}
$$

This completes the proof.

\section{Acknowledgements}

The authors are grateful to an anonymous referee for comments and suggestions. This research was partially supported by the bilateral France-Lithuania scientific project 'Gilibert'. The first author was partially supported by NATO grant PST.EAP.CLG 980599; the second author was partially supported by the Lithuanian State Science and Studies Foundation grant T-10/06.

\section{References}

Baccelli, F. And Brémaud, P. (1994). Elements of Queueing Theory: Palm Martingale Calculus and Stochastic Recurrences. Springer, New York.

Borovkov, A. A. (1976). Stochastic Processes in Queueing Theory. Springer, New York.

Daley, D. J. ANd Vesilo, R. A. (1997). Long range dependence of point processes, with queueing examples. Stoch. Process. Appl. 70, 265-282.

Daley, D. J. AND VesiLo, R. A. (2000). Long range dependence of inputs and outputs of some classical queues. Fields Inst. Commun. 28, 179-186.

Franken, P., König, D., Arndt, U. And Schmidt, V. (1981). Queues and Point Processes. Springer, Berlin.

HeAth, D., ReSnick, S. AND SAmOrodnitsky, G. (1998). Heavy tails and long range dependence in ON/OFF processes and associated fluid models. Math. Operat. Res. 23, 145-165.

Smith, W. L. (1958). Renewal theory and its ramifications. J. R. Statist. Soc. B 20, 243-302.

Willinger, W., Taqqu, M. S., Sherman, R. and Wilson, D. V. (1997). Self-similarity through high-variability: statistical analysis of Ethernet LAN traffic at the source level. IEEE/ACM Trans. Networking 5, 71-86. 